\title{
Development of Near Infrared-Fluorescent Nanophosphors and Applications for Cancer Diagnosis and Therapy
}

\author{
Tamotsu Zako, ${ }^{1,2}$ Hiroshi Hyodo, ${ }^{3,4,5}$ Kosuke Tsuji, $^{3}$ Kimikazu Tokuzen, ${ }^{3}$ \\ Hidehiro Kishimoto, ${ }^{5,6}$ Masaaki Ito, ${ }^{7}$ Kazuhiro Kaneko, ${ }^{8}$ Mizuo Maeda, ${ }^{1,2}$ \\ and Kohei Soga ${ }^{3,4,5}$ \\ ${ }^{1}$ Bioengineering Laboratory, RIKEN Institute, 2-1 Hirosawa, Wako, Saitama 351-0198, Japan \\ ${ }^{2}$ Nano-Scale Science \& Technology Research Group, RIKEN Institute, 2-1 Hirosawa, Wako, Saitama 351-0198, Japan \\ ${ }^{3}$ Department of Material Science and Technology, Tokyo University of Science, 2641 Yamazaki, Noda, Chiba 278-8510, Japan \\ ${ }^{4}$ Polyscale Technology Research Center, Tokyo University of Science, 2641 Yamazaki, Noda, Chiba 278-8510, Japan \\ ${ }^{5}$ Center for Technologies against Cancer, Tokyo University of Science, 2641 Yamazaki, Noda, Chiba 278-8510, Japan \\ ${ }^{6}$ Research Institute for Biological Sciences, Tokyo University of Science, 2669 Yamazaki, Noda, Chiba 278-0022, Japan \\ ${ }^{7}$ Department of Colorectal and Pelvic Surgery, National Cancer Center Hospital East, Kashiwanoha 6-5-1, Kashiwa, \\ Chiba 277-8577, Japan \\ ${ }^{8}$ Division of Digestive Endoscopy and Gastrointestinal Oncology, National Cancer Center Hospital East, Kashiwanoha \\ 6-5-1, Kashiwa, Chiba 277-8577, Japan
}

Correspondence should be addressed to Tamotsu Zako, zako@riken.jp and Kohei Soga, mail@ksoga.com

Received 1 November 2009; Revised 25 December 2009; Accepted 5 March 2010

Academic Editor: Huisheng Peng

Copyright (C) 2010 Tamotsu Zako et al. This is an open access article distributed under the Creative Commons Attribution License, which permits unrestricted use, distribution, and reproduction in any medium, provided the original work is properly cited.

\begin{abstract}
The use of near infrared (NIR) light for biomedical photonics in the wavelength region between 800 and $2000 \mathrm{~nm}$, which is called "biological window", has received particular attention since water and biological tissues have minimal optical loss due to scattering and absorption as well as autofluorescence in this region. Recent development of InGaAs CCD enables observations in this wavelength region. In the present paper, we report development of $\mathrm{Yb}$ and Er-doped yttrium oxide nanoparticles $\left(\mathrm{Y}_{2} \mathrm{O}_{3}: \mathrm{YbEr}\right.$ NP) which show strong NIR emission under NIR excitation (NIR-NIR emission). We also demonstrate that NIR emission can be observed through swine colon wall. Based on these results, we propose a possible application of $\mathrm{Y}_{2} \mathrm{O}_{3}: \mathrm{YbEr}$-NP for cancer diagnosis and therapy using NIR-NIR imaging system. Our results also suggest potential applications of $\mathrm{Y}_{2} \mathrm{O}_{3}$ :YbEr-NP for noninvasive detection of various diseases.
\end{abstract}

\section{Introduction}

Bioimaging technique has received particular attention as an essential tool in the field of biomedical research through the observation of biological phenomena both in vivo and in vitro. The use of near infrared (NIR) light in the wavelength region between 800 and $2000 \mathrm{~nm}$ for biomedical photonics attracts great interest because this region is a so-called "biological window", where water and biological tissues have minimal absorbance and autofluorescence. As shown in the loss spectrum of human skin [1] (see Figure 1 of Supplementary Material available online at doi:10.1155/2010/491471.), one can expect the lowest loss of the spectrum within the above region.
Recently, upconverting (UC) phosphors (UCPs) have been used for bioimaging (Figure 1) [2-8]. UCPs are ceramic materials containing rare earth ions. The materials can absorb IR radiation and upconvert it to emit visible light by stepwise excitation among discrete energy levels of the rare earth ions (NIR-VIS imaging) [9]. For example, yttrium oxide $\left(\mathrm{Y}_{2} \mathrm{O}_{3}\right)$ matrix containing several atomic \% of erbium (Er) exhibits upconversion emission at $550 \mathrm{~nm}$ (green) and $660 \mathrm{~nm}$ (red) following excitation at $980 \mathrm{~nm}$. The advantage of NIR-VIS bioimaging is that NIR light can penetrate deeper into tissues due its lower scattering.

The wavelength for biomedical photonics has been limited due to the use of the silicon-based CCD. The observation wavelength is limited to at most $1100 \mathrm{~nm}$ due to the band 
gap of silicon. In recent years, however, the InGaAs CCD which can cover wavelength between 800 and $2200 \mathrm{~nm}$ has become available. Considering various advantages of the NIR window, the time is ideal for the development of phosphors to emit fluorescence in this region.

Rare-earth doped ceramics can be a good candidate, since these are known to emit efficient fluorescence in the NIR wavelength region by NIR excitation. For example, the most representative solid state laser material Nd:YAG (Nd-doped yttrium aluminum garnet) can emit light with a wavelength of 1064-nm with 800-nm excitation [10]. Erdoped silicate glass fibers are used to amplify the signal of long-distance fiber optical communication by emitting 1550-nm fluorescence with $980-\mathrm{nm}$ excitation [11]. The authors have previously reported that Er-doped yttrium oxide nanoparticles $\left(\mathrm{Y}_{2} \mathrm{O}_{3}\right.$ :Er-NP) showed NIR fluorescence $(1550 \mathrm{~nm})$ with NIR excitation [12]. The advantage of this NIR-NIR imaging is that both excitation and emission light can penetrate deep into/from tissues, which enables imaging of the target inside the tissues (Figure 1).

In this study, we report a development of $\mathrm{Yb}$ and $\mathrm{Er}-$ doped yttrium oxide nanoparticles $\left(\mathrm{Y}_{2} \mathrm{O}_{3}: \mathrm{YbEr}-\mathrm{NP}\right)$, which possess higher NIR emission than $\mathrm{Y}_{2} \mathrm{O}_{3}$ : $\mathrm{Er}-\mathrm{NP}$. $\mathrm{Yb}^{3+}$ was added as a so-called "sensitizer" to increase the NIR emission. Since $\mathrm{Yb}^{3+}$ has much larger absorption efficiency and the excitation energy can be efficiently transferred to $\mathrm{Er}^{3+}$ in case of upconversion phosphors [9], we added the $\mathrm{Yb}^{3+}$ codopant as a sensitizer expecting the same effect for the $1550 \mathrm{~nm}$ NIR emission of $\mathrm{Er}^{3+}$. We also demonstrated for the first time that NIR emission could be observed even through the swine colon wall. Based on this observation, we propose possible new NIR-NIR biophotonics applications for cancer diagnosis and therapy using $\mathrm{Y}_{2} \mathrm{O}_{3}: \mathrm{YbEr}-\mathrm{NP}$, especially for resection surgery of colon cancer.

\section{Material and Methods}

2.1. Materials. $\mathrm{Y}\left(\mathrm{NO}_{3}\right)_{3} \cdot 6 \mathrm{H}_{2} \mathrm{O}(99.99 \%$ purity) and Urea $(99.0 \%$ purity) were purchased from Kanto Chemicals (Tokyo, Japan). $\mathrm{Er}\left(\mathrm{NO}_{3}\right)_{3} \cdot 5 \mathrm{H}_{2} \mathrm{O}$ ( $>99 \%$ purity), $\mathrm{Yb}\left(\mathrm{NO}_{3}\right)_{3}$. $5 \mathrm{H}_{2} \mathrm{O}\left(99.9 \%\right.$ purity) and $\mathrm{Na}_{2} \mathrm{CO}_{3}$ (99\% purity) were obtained from Kojundo Chemical Laboratory (Saitama, Japan).

2.2. Preparation of NIR Biophotonic Nanoparticles. $\mathrm{Y}_{2} \mathrm{O}_{3}$ : $\mathrm{YbEr}-\mathrm{NP}$ were prepared by the homogeneous precipitation method as used for preparation of upconversion nanoparticles [13]. Twenty mmol/L Y $\left(\mathrm{NO}_{3}\right)_{3}, 0.2 \mathrm{mmol} / \mathrm{L} \mathrm{Yb}\left(\mathrm{NO}_{3}\right)_{3}$, and $0.2 \mathrm{mmol} / \mathrm{L} \mathrm{Er}\left(\mathrm{NO}_{3}\right)_{3}$ were dissolved in $200 \mathrm{~mL}$ purified water, mixed with $100 \mathrm{~mL}$ of $4 \mathrm{~mol} / \mathrm{L}$ Urea solution, and stirred for 1 hour at $100^{\circ} \mathrm{C}$. The obtained precipitates were separated by centrifugation, and dried at $80^{\circ} \mathrm{C}$ for 12 hours. The hydroxide or hydroxyl carbonated precursors were calcinated at $1200^{\circ} \mathrm{C}$ for 60 minutes in an electric furnace to convert them into anhydrous crystalline $\mathrm{Y}_{2} \mathrm{O}_{3}$ nanoparticles doped with $\mathrm{Yb}$ and Er.

2.3. Characterization of NIR Biophotonic Nanoparticles. The prepared $\mathrm{Y}_{2} \mathrm{O}_{3}: \mathrm{YbEr}-\mathrm{NP}$ were provided for characterization

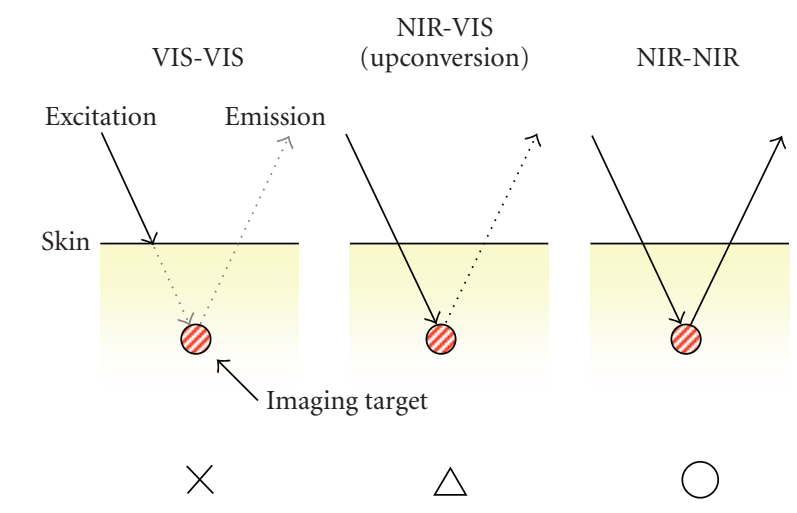

FIgURE 1: Advantage of NIR-NIR imaging system. Near infrared (NIR) emission by NIR excitation is observed using a NIR-NIR system. Due to weaker scattering and absorption, NIR light can penetrate deeper into/from tissues. In contrast, excitation light in the visible (VIS) region cannot reach the imaging target in tissues in the conventional VIS-VIS imaging. In upconversion (NIR-VIS) imaging, although NIR excitation light can reach its target in tissues, only a weak VIS emission can be obtained.

using FE-SEM (S-4200, Hitachi Ltd., Tokyo, Japan) and XRD (XRD-6100, Shimadzu, Kyoto, Japan) with $\mathrm{CuK} \alpha$ radiation.

Optical absorption spectra were observed using a spectrometer (U-4000, Hitachi Ltd., Tokyo, Japan) equipped with an integrating sphere. The loss spectrum of the swine colon was also observed using the same equipment and sandwiching a slice of the colon (thickness: $250-330 \mu \mathrm{m}$ ) between two glass slides. The loss spectra were measured in a normal mode without using the integrating sphere.

Fluorescence spectra of $\mathrm{Y}_{2} \mathrm{O}_{3}$ : $\mathrm{YbEr}-\mathrm{NP}$ and $\mathrm{Y}_{2} \mathrm{O}_{3}$ :Er-NP were recorded using a spectrometer (AvaSpec-NIR256-1.7, Avantes, Eerbeek, Netherlands) under an excitation of 980$\mathrm{nm}$ and a laser diode (LD, SLI-CW-9MM-C1-980-1M-PD, Semiconductor Laser International Corp., USA).

2.4. NIR Imaging System. NIR-NIR imaging was carried out using the NIR imaging system, consisting of a fiber pigtail laser diode at $980 \mathrm{~nm}$ with $2 \mathrm{~W}$ power (LU0975T050, Lumics, Berlin, Germany), a laser scanner (VM500+, GSI Group, Massachusetts, USA) for planer irradiation of the excitation light, and InGaAs CCD camera (NIR-300PGE, VDS Vosskühler, Osnabrück, Germany) for detection of the NIR fluorescence between 1100 and $1600 \mathrm{~nm}$.

2.5. NIR Imaging Inside Swine Colon. In order to demonstrate that NIR light under NIR excitation can be observed through the colon wall, a tablet of $\mathrm{Y}_{2} \mathrm{O}_{3}: \mathrm{YbEr}-\mathrm{NP}$ with a diameter of $3 \mathrm{~mm}$ and a length of $6 \mathrm{~mm}$ was formed by mixing $\mathrm{Y}_{2} \mathrm{O}_{3}$ : $\mathrm{YbEr}-\mathrm{NP}$ with a conventional dental composite resin (Fuji I, GC, Tokyo, Japan). An endoscopic clip [14] (Olympus, Tokyo, Japan) painted with $\mathrm{Y}_{2} \mathrm{O}_{3}$ :YbEr-NPcontaining paint (NIR clip) was also prepared in order to demonstrate that NIR light from the clip can also be observed through colon wall. After fixing the NIR clip in the mucosal side (inside) of a piece of the tubular swine colon, we observed the colon using the NIR-NIR imaging system. It is 


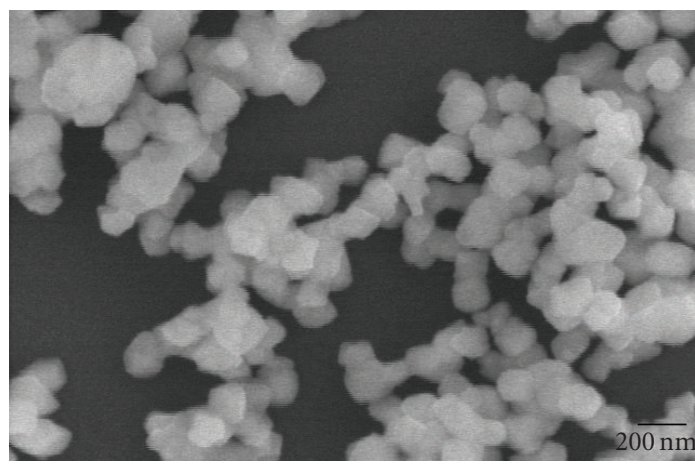

(a)

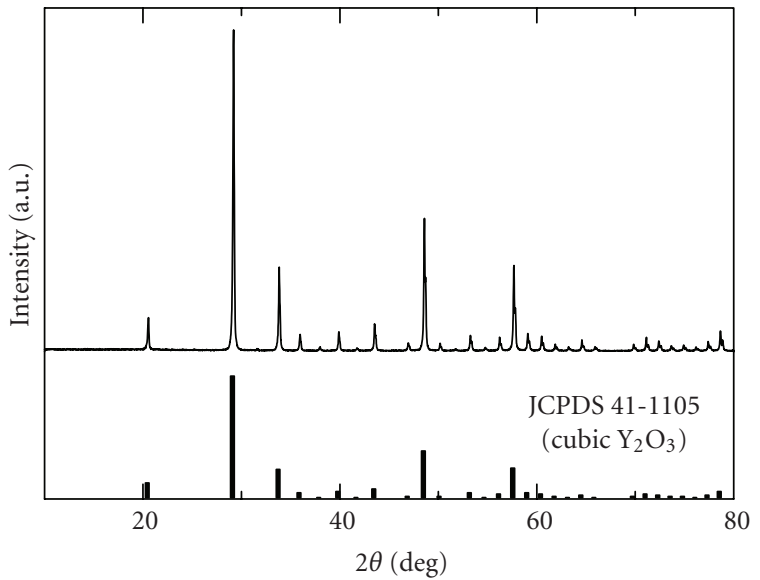

(b)

FIgURE 2: Characterization of NIR biophotonic nanoparticles (a) FE-SEM images of $\mathrm{Y}_{2} \mathrm{O}_{3}$ :YbEr-NP synthesized by homogeneous precipitation and calcination at $1200^{\circ} \mathrm{C}$ for 60 minutes. The particle size was approximately $130 \pm 25 \mathrm{~nm}$. The scale bar represents $200 \mathrm{~nm}$. (b) XRD patterns of $\mathrm{Y}_{2} \mathrm{O}_{3}: \mathrm{YbEr}-\mathrm{NP}$.

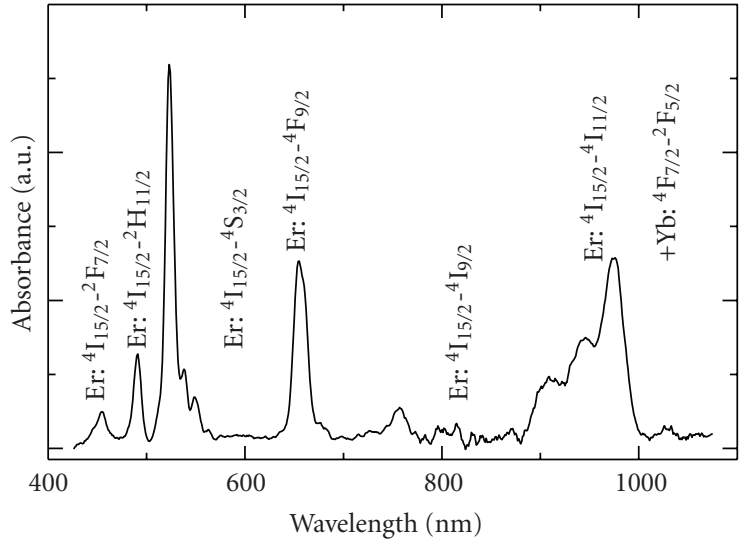

(a)

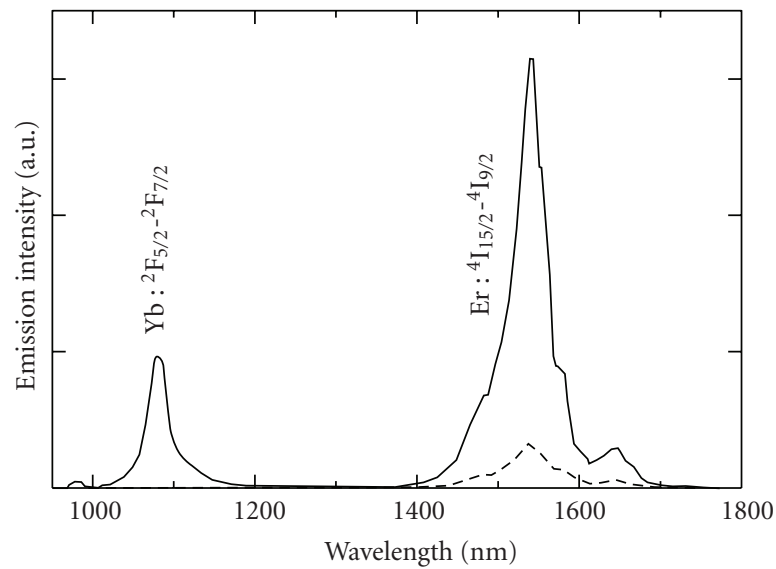

(c)

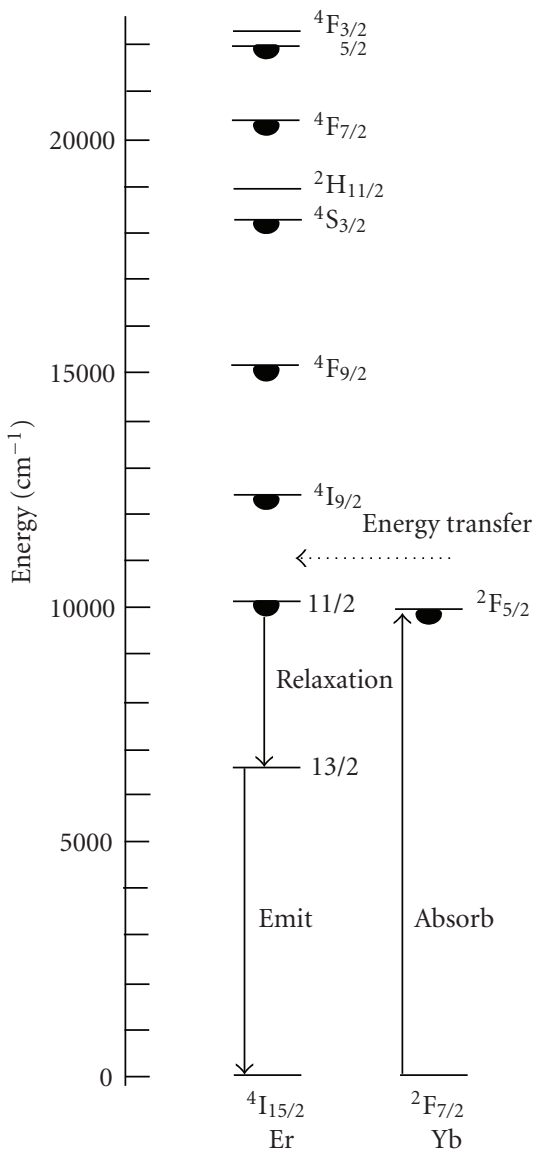

(b)

Figure 3: (a) Absorption spectrum of $\mathrm{Y}_{2} \mathrm{O}_{3}: \mathrm{YbEr}-\mathrm{NP}$. (b) Energy level diagram of $\mathrm{Y}_{2} \mathrm{O}_{3}: \mathrm{YbEr}-\mathrm{NP}$. (c) Fluorescence spectra of $\mathrm{Y}_{2} \mathrm{O}_{3}: \mathrm{YbEr} \mathrm{NP}$ (solid line) and $\mathrm{Y}_{2} \mathrm{O}_{3}$ :Er-NP (dot line). The fluorescence was measured under an excitation wavelength of $980 \mathrm{~nm}$. 


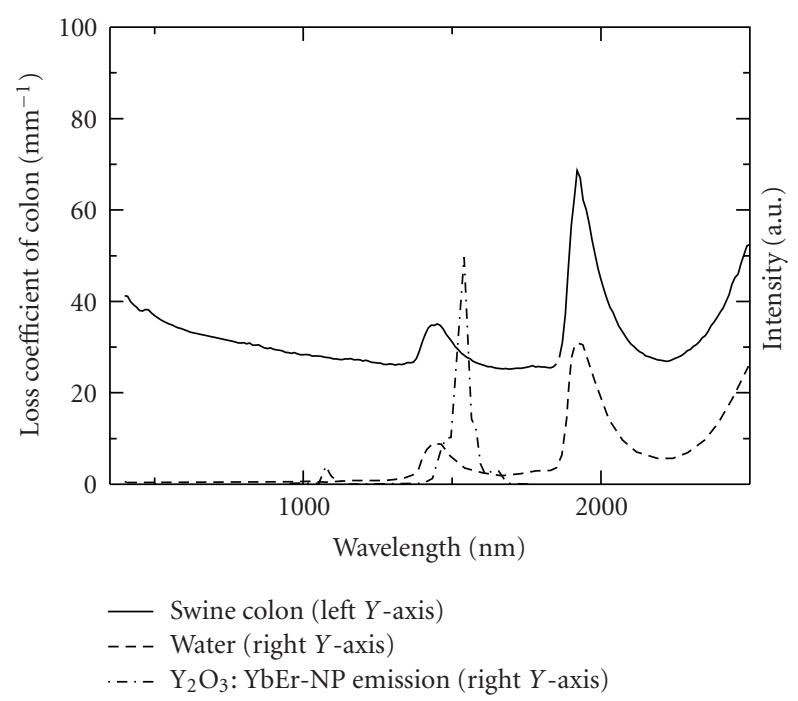

Figure 4: Loss spectrum of a sliced sample of swine colon (solid line). The absorption peak at $1420 \mathrm{~nm}$ corresponds to the second harmonic of the infrared absorption of water and depends on the water content. The water absorption spectrum is inserted with a dashed line. The emission spectrum of $\mathrm{Y}_{2} \mathrm{O}_{3}: \mathrm{YbEr}-\mathrm{NP}$ is also incorporated with a chain line.

expected that the painted portion of these endoscopic clips will be observed through the colon wall from the serosal side (outside) of the colon.

\section{Results and Discussion}

3.1. Characterization of NIR Biophotonic Nanoparticles. Figure 2(a) shows FE-SEM images of $\mathrm{Y}_{2} \mathrm{O}_{3}: \mathrm{YbEr}$-NP synthesized by homogeneous precipitation and calcination at $1200^{\circ} \mathrm{C}$ for 60 minutes. The particle size was approximately $130 \pm 25 \mathrm{~nm}$. Figure 2(b) shows the XRD pattern of $\mathrm{Y}_{2} \mathrm{O}_{3}: \mathrm{YbEr}-\mathrm{NP}$. The sample was confirmed to be single-phase $\mathrm{Y}_{2} \mathrm{O}_{3}$ since all of the peaks were identified as those of cubic $\mathrm{Y}_{2} \mathrm{O}_{3}$ (JCPDS 41-1105).

3.2. Absorption and Fluorescence Spectra. Absorption and fluorescence spectra of $\mathrm{Y}_{2} \mathrm{O}_{3}: \mathrm{YbEr}-\mathrm{NP}$ are shown in Figure 3. $\mathrm{Yb}^{3+}$ was added as a so-called "sensitizer" for increasing the absorption efficiency of the excitation light at $980 \mathrm{~nm}$ in this study. In the absorption spectrum (Figure 3(a)), a strong absorption band of $\mathrm{Yb}^{3+}$ was observed. The absorbed excitation light at $980 \mathrm{~nm}$ was mainly absorbed by $\mathrm{Yb}^{3+}$ and the excitation energy transfers to $\mathrm{Er}^{3+}$ to emit the NIR fluorescence at $1550 \mathrm{~nm}$, as shown in Figure 3(b). The absorption and florescence schemes are well known in the field of optical communication and the phenomenon has been well understood $[10,11]$. Figure $3(\mathrm{c})$ shows that the NIR emission of $\mathrm{Y}_{2} \mathrm{O}_{3}: \mathrm{YbEr}-\mathrm{NP}$ is much higher than that of $\mathrm{Y}_{2} \mathrm{O}_{3}$ :Er-NP, indicating that codoping of $\mathrm{Yb}^{3+}$ is also effective to enhance NIR emission.
Figure 4 shows the loss spectrum of the slice of swine colon. The spectrum was obtained by deducting the spectrum due to a thickness of $250 \mu \mathrm{m}$ from that of $330 \mu \mathrm{m}$ to yield the net loss due to a swine colon thickness of $110 \mu \mathrm{m}$. The spectrum is divided by the corresponding thickness to make it a coefficient spectrum. A water absorption spectrum as well as the emission spectrum of $\mathrm{Y}_{2} \mathrm{O}_{3}$ : $\mathrm{YbEr}-\mathrm{NP}$, were also coplotted. There are absorption band peaks at $1420 \mathrm{~nm}$, which are due to the second harmonic absorption of the $\mathrm{O}$ $\mathrm{H}$ stretching vibration in water molecules. In the spectrum, the fluorescence spectrum is super imposed. Although the fluorescence and the absorption bands overlap, the tail of the fluorescence is still out of the absorption band and one can expect observation of the fluorescence through the colon wall. It appeared better to select phosphors which could emit fluorescence avoiding the water absorption at $1420 \mathrm{~nm}$. The development of the phosphors that can emit NIR light at different wavelength by doping different rare-earth ions such as $\mathrm{Nd}$, Pr or $\mathrm{Tm}$ is now in progress.

3.3. NIR Imaging. Figure 5(a) shows images of the $\mathrm{Y}_{2} \mathrm{O}_{3}$ :YbEr-NP tablet set in a tubular swine colon. The tablet emission could be clearly observed even through the colon wall. This result indicates that the NIR excitation light and the NIR emission from $\mathrm{Y}_{2} \mathrm{O}_{3}: \mathrm{YbEr}-\mathrm{NP}$ is strong enough to penetrate the colon wall.

In an effort to show the applicability of $\mathrm{Y}_{2} \mathrm{O}_{3}: \mathrm{YbEr}-\mathrm{NP}$ in cancer therapy, NIR imaging of $\mathrm{Y}_{2} \mathrm{O}_{3}$ :YbEr-NP-coated medical clips and $\mathrm{Y}_{2} \mathrm{O}_{3}: \mathrm{YbEr}-\mathrm{NP}$ solution injected from the mucosal side (inside) of the colon were carried out. The clips used in this experiment are commercially available for endoscopic therapy and can be easily employed to mark the part of cancer using a conventional endoscopy system. The coating was applied onto the plastic part of the clip. Figure 5(b) shows the NIR imaging $\mathrm{Y}_{2} \mathrm{O}_{3}$ :YbEr-NP-coated clips and those set inside of the swine colon under NIR excitation. Although the coating was as thin as several tens of $\mu \mathrm{m}$, the NIR fluorescence was clearly observed and was comparable to the case of the tablets.

NIR imaging of $\mathrm{Y}_{2} \mathrm{O}_{3}$ : $\mathrm{YbEr}-\mathrm{NP}$ solution injected inside the colon was also carried out. As shown in Figure 5(c), NIR emission from $\mathrm{Y}_{2} \mathrm{O}_{3}$ : $\mathrm{YbEr}-\mathrm{NP}$ injected in the other side of the colon wall was clearly observed. This result suggests that $\mathrm{Y}_{2} \mathrm{O}_{3}: \mathrm{YbEr}-\mathrm{NP}$ can be used as a substitution for tattoo (black ink) solution which is usually used in cancer therapy as described below. Since tattoo solution is usually injected at both ends of tumor region before laparoscopic surgery, $\mathrm{Y}_{2} \mathrm{O}_{3}$ :YbEr-NP solution was also injected at two points.

\subsection{Possible Applications of NIR Photonic Nanomaterials for} Cancer Diagnosis and Therapy. The spectroscopic properties of swine colon and the development and demonstrative work using $\mathrm{Y}_{2} \mathrm{O}_{3}: \mathrm{YbEr}-\mathrm{NP}$ suggest a great potential of NIR-NIR photonic nanomaterials for cancer therapy. For example, this technology can be applied to the intraoperative recognition of the tumor site in laparoscopic surgery for the gastrointestinal cancer (Figures 6(a) and 6(b)). Tattooing 


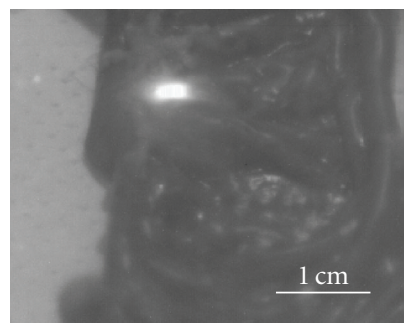

(a)
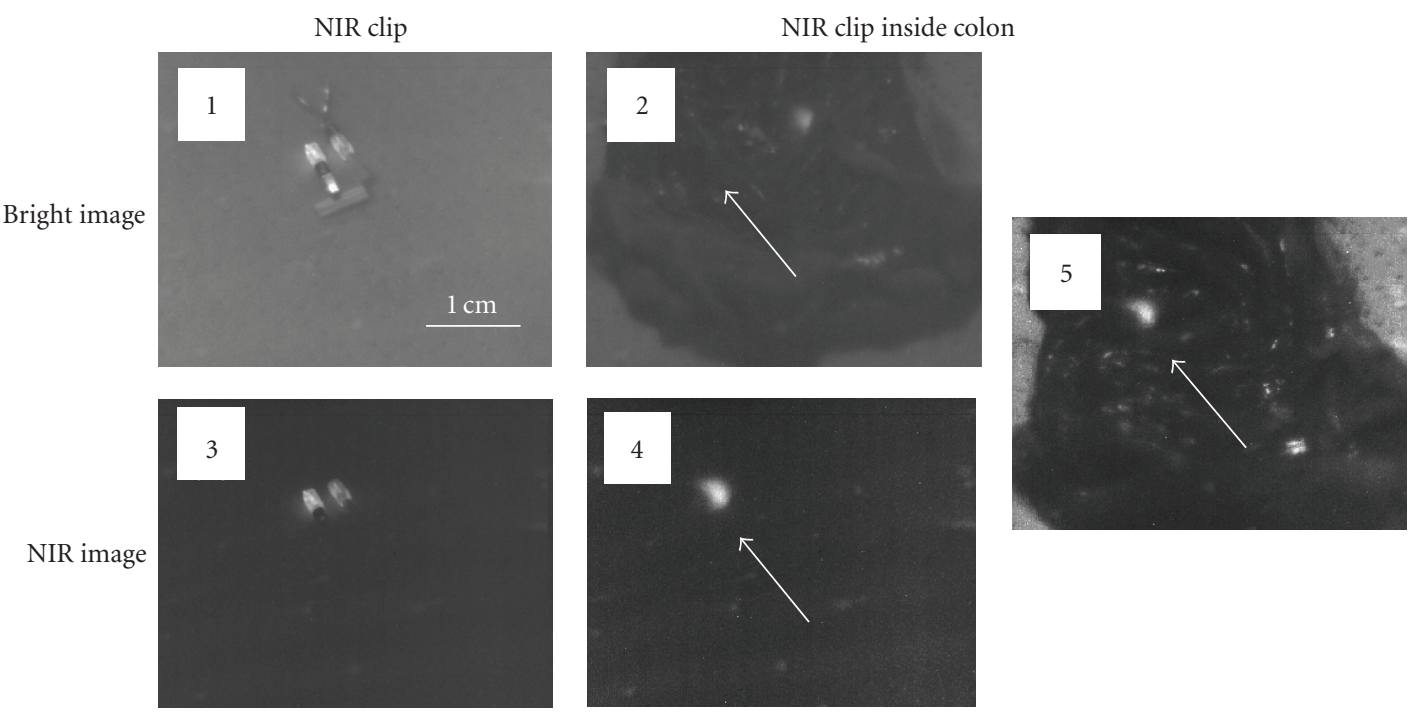

(b)

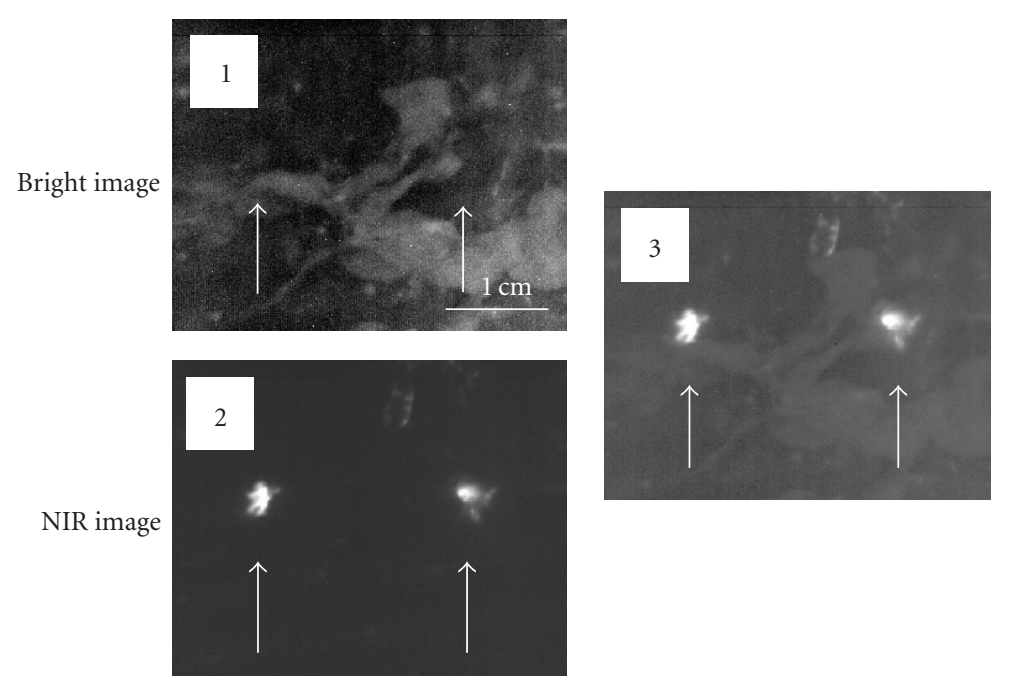

(c)

Figure 5: NIR imaging (a) $\mathrm{Y}_{2} \mathrm{O}_{3}$ :YbEr-NP tablet set in the tubular sample of swine colon. Hybrid image of bright-field and NIR fluorescence is shown. (b) Bright-filed $(1,2)$ and NIR fluorescence $(3,4)$ images of $\mathrm{Y}_{2} \mathrm{O}_{3}$ :YbEr-NP-coated clips $(1,3)$ and those set inside the swine colon $(2,4)$. Observations of (2) and (4) are from outside of the colon. Hybrid image of the $\mathrm{Y}_{2} \mathrm{O}_{3}$ : YbEr-NP-coated clips set in the swine colon (5) are shown. Arrows in (2), (4), and (5) show the position of the clip inside swine colon. (c) $\mathrm{Y}_{2} \mathrm{O}_{3}: \mathrm{YbEr}-\mathrm{NP}$ solution (10 mg/mL) injected from the reverse side of the swine colon. Bright-field (1), NIR fluorescence (2) and hybrid (3) images are shown. Observations are from outside of the colon. Arrows show the injection position of $\mathrm{Y}_{2} \mathrm{O}_{3}$ : $\mathrm{YbEr}-\mathrm{NP}$ solution inside swine colon. 
1. Endoscopic cancer detection and fixation NIR clip

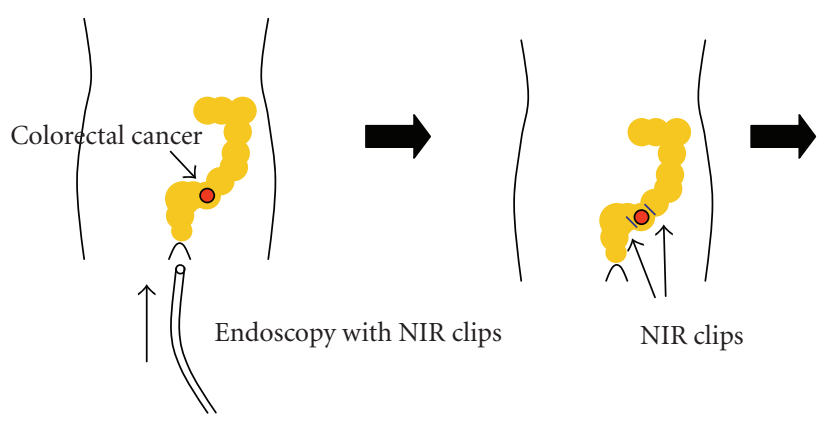

Tattooing (current procedure)

Colorectal cancer

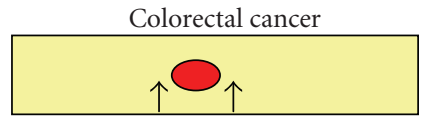

Anal side

Tattoo injection

Oral side
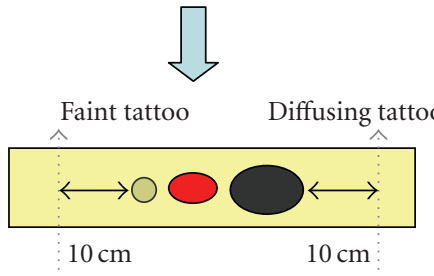

Spread resection
2. Minimum colon resection using NIR-NIR imaging system

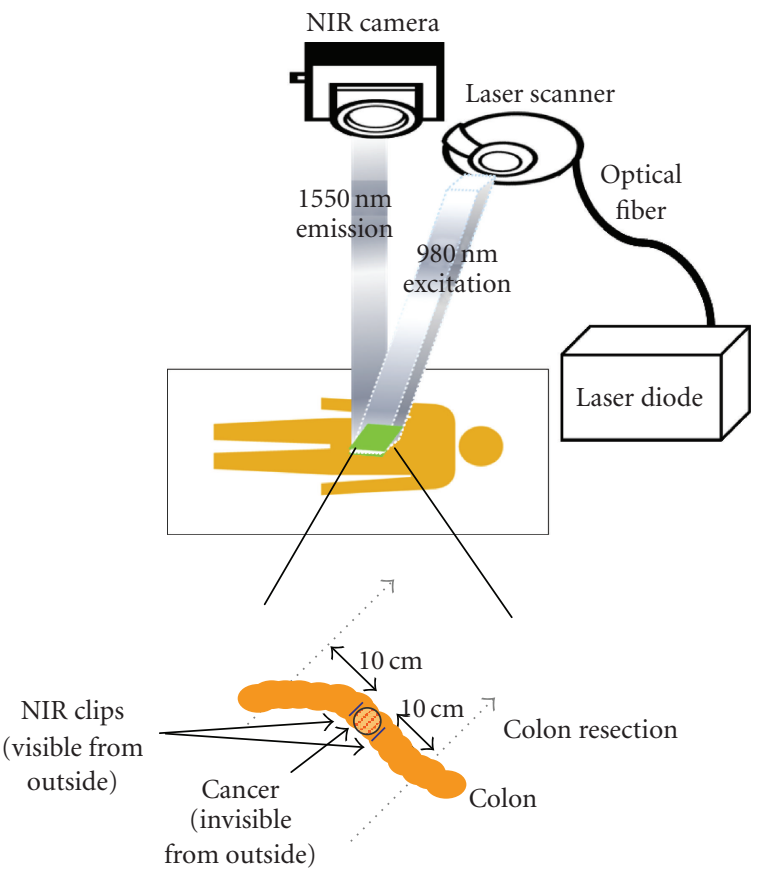

(a)

NIR clip (proposed procedure)

Colorectal cancer

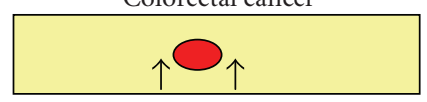

Fix NIR clip
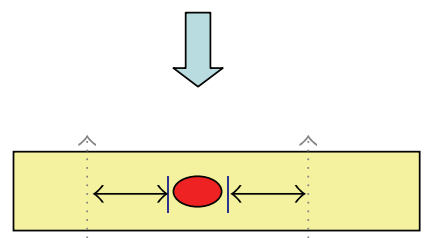

$10 \mathrm{~cm} \quad 10 \mathrm{~cm}$

Minimum resection

(b)

FIgURE 6: Possible application of NIR photonic nanomaterials for cancer therapy. (a) Usage of $\mathrm{Y}_{2} \mathrm{O}_{3}$ :YbEr-NP-coated clips (NIR clips) for colorectal cancer surgery. (1) After endoscopic detection of colorectal cancer, NIR clips are fixed to mark cancer sites using endoscopy. (2) Cancer surgery using NIR-NIR imaging system. Using this new imaging system, we will be able to determine the proper resection margins (normally $10 \mathrm{~cm}$ from the cancer site) for curative resection during surgery. (b) Advantage of proposed NIR clip procedure against current procedure (tattooing). NIR clips enable better recognition of cancer sites, which leads to minimum colon resection.

into the submucosal layer of the colon is generally performed in laparoscopic surgery, which sometimes leads to difficulty in recognition of cancer site due to faint tattoo and diffused tattoo, which causes spread resection of the colon (Figure 6(b)) [15]. Figure 6(a) shows our proposed procedure using NIR clips. After endoscopic detection of colorectal cancer, NIR clips are fixed to mark cancer site using endoscopy. Cancer site can be recognized through the serosa of the intestinal wall by NIR fluorescence from the NIR clips fixed inside the colon during cancer surgery using NIR-NIR imaging system. Using this new imaging system, we will be able to determine the proper resection margins (normally $10 \mathrm{~cm}$ from the cancer site) for curative resection during surgery, which is much more advantageous compared with the current procedure using tattoo (Figure 6(b)).

$\mathrm{Y}_{2} \mathrm{O}_{3}$ :YbEr-NP can also be used for caner diagnostics. Previously we have demonstrated tumor cell-targeted upconversion imaging using $\mathrm{Y}_{2} \mathrm{O}_{3}$ :Er-NP modified with cyclic 
arginine-glycine-aspartic acid (RGD) peptide as a specific probe for tumor cell detection [7]. The RGD peptide strongly binds to integrin $\alpha_{v} \beta_{3}$, whose expression is significantly upregulated in invasive tumor cells of certain cancer types (glioblastoma, melanoma, breast, ovarian, and prostate cancers, and in almost all tumor vasculature), but not in quiescent endothelium and normal tissues $[16,17]$. Thus, modification of $\mathrm{Y}_{2} \mathrm{O}_{3}: \mathrm{YbEr}-\mathrm{NP}$ with cyclic RGD peptide will also be useful for the development of a tumor celltargeted NIR-NIR imaging probe. Successful observation of NIR emission from $\mathrm{Y}_{2} \mathrm{O}_{3}$ :YbEr-NP solution injected inside the colon (Figure 5(c)) supports the idea that targeting and detection of cancer sites in colon using $\mathrm{Y}_{2} \mathrm{O}_{3}: \mathrm{YbEr}-\mathrm{NP}$ are possible. Research along this line is currently in progress. Our results also suggest that probe-modified $\mathrm{Y}_{2} \mathrm{O}_{3}$ : $\mathrm{YbEr}-\mathrm{NP}$ could be used for noninvasive detection of various diseases.

Cell toxicity is another important issue when considering probes for use in bioimaging. Previous studies showed that $\mathrm{Y}_{2} \mathrm{O}_{3}$ and $\mathrm{Er}^{3+}$-doped $\mathrm{Y}_{2} \mathrm{O}_{3}$ nanoparticles were nontoxic to cultured cell $[6,18]$. Since the chemical properties of $\mathrm{Yb}^{3+}$ are similar to those of $\mathrm{Er}^{3+}$ [19], it is plausible that $\mathrm{Yb}^{3+}$ and $\mathrm{Er}^{3+}$-doped $\mathrm{Y}_{2} \mathrm{O}_{3}$ nanoparticles also are nontoxic. However, further studies on biocompatibility such as inflammation assays and long-term toxicity assays using animal models are important for their medical application.

\section{Conclusion}

The use of near infrared (NIR) light in the wavelength region between 800 and $2000 \mathrm{~nm}$ for biomedical photonics attracts great interest. This region is a so-called "biological window", where water and biological tissues have minimal absorbance and autofluorescence. In the present study, we report high NIR emission under NIR excitation (NIR-NIR emission) of $\mathrm{Yb}$ and Er-doped yttrium oxide nanoparticles $\left(\mathrm{Y}_{2} \mathrm{O}_{3}\right.$ : $\mathrm{YbEr}$ $\mathrm{NP}$ ), and propose a possible NIR-NIR biophotonic application using $\mathrm{Y}_{2} \mathrm{O}_{3}$ : $\mathrm{YbEr}-\mathrm{NP}$ for cancer diagnosis and therapy based on demonstrative experiments. Observations of NIR emission through swine colon wall support our idea that NIR-NIR biophotonic nanomaterials can be used for cancer diagnosis and therapy.

\section{Acknowledgments}

The authors thank Professor Atsuo Yasumori for the XRD measurement and Dr. Karin Sörgjerd for helpful comments. This work is financially supported by Industrial Technology Research Grant Program from New Energy and Industrial Technology Development Organization (NEDO) of Japan (Tamotsu Zako).

\section{References}

[1] R. R. Anderson and J. A. Parrish, "The optics of human skin," Journal of Investigative Dermatology, vol. 77, no. 1, pp. 13-19, 1981.

[2] M. Kamimura, D. Miyamoto, Y. Saito, K. Soga, and Y. Nagasaki, "Design of poly(ethylene glycol)/streptavidin coimmobilized upconversion nanophosphors and their application to fluorescence biolabeling," Langmuir, vol. 24, no. 16, pp. 88648870, 2008.

[3] S. F. Lim, R. Riehn, W. S. Ryu, et al., "In vivo and scanning electron microscopy imaging of upconverting nanophosphors in Caenorhabditis elegans," Nano Letters, vol. 6, no. 2, pp. 169174, 2006.

[4] P. N. Prasad, "Emerging opportunities at the interface of photonics, nanotechnology and biotechnology," Molecular Crystals and Liquid Crystals, vol. 415, pp. 1-7, 2004.

[5] S. Sivakumar, P. R. Diamente, and F. C. van Veggel, "Silicacoated $\mathrm{Ln}^{3+}$-Doped $\mathrm{LaF}_{3}$ nanoparticles as robust down- and upconverting biolabels," Chemistry: A European Journal, vol. 12, no. 22, pp. 5878-5884, 2006.

[6] T. Zako, H. Nagata, N. Terada, M. Sakono, K. Soga, and M. Maeda, "Improvement of dispersion stability and characterization of upconversion nanophosphors covalently modified with PEG as a fluorescence bioimaging probe," Journal of Materials Science, vol. 43, no. 15, pp. 5325-5330, 2008.

[7] T. Zako, H. Nagata, N. Terada, et al., "Cyclic RGD peptidelabeled upconversion nanophosphors for tumor cell-targeted imaging," Biochemical and Biophysical Research Communications, vol. 381, no. 1, pp. 54-58, 2009.

[8] H. J. Zijlmans, J. Bonnet, J. Burton, et al., "Detection of cell and tissue surface antigens using up-converting phosphors: a new reporter technology," Analytical Biochemistry, vol. 267, no. 1, pp. 30-36, 1999.

[9] F. Auzel, "Upconversion and anti-stokes processes with $\mathrm{f}$ and $\mathrm{d}$ ions in solids," Chemical Reviews, vol. 104, no. 1, pp. 139-173, 2004.

[10] R. C. Powell, Physics of Solid State Laser Materials, Springer, New York, NY, USA, 1998.

[11] S. Sudo, Optical Fiber Amplifiers: Materials, Devices, and Applications, Artech House, Norwood, Mass, USA, 1997.

[12] N. Venkatachalam, Y. Okumura, K. Soga, R. Fukuda, and T. Tsuji, "Bioimaging of $\mathrm{M} 1$ cells using ceramic nanophosphors: synthesis and toxicity assay of $\mathrm{Y}_{2} \mathrm{O}_{3}$ nanoparticles," Journal of Physics: Conference Series, vol. 191, Article ID 012002, 2009.

[13] N. Venkatachalam, Y. Saito, and K. Soga, "Synthesis of $\mathrm{Er}^{3+}$ doped $\mathrm{Y}_{2} \mathrm{O}_{3}$ nanophosphors," Journal of the American Ceramic Society, vol. 92, no. 5, pp. 1006-1010, 2009.

[14] G. S. Raju and L. Gajula, "Endoclips for GI endoscopy," Gastrointestinal Endoscopy, vol. 59, no. 2, pp. 267-279, 2004.

[15] K. L. Lane, R. Vallera, K. Washington, and M. R. Gottfried, "Endoscopic tattoo agents in the colon: tissue responses and clinical implications," American Journal of Surgical Pathology, vol. 20, no. 10, pp. 1266-1270, 1996.

[16] J. D. Hood and D. A. Cheresh, "Role of integrins in cell invasion and migration," Nature Reviews Cancer, vol. 2, no. 2, pp. 91-100, 2002.

[17] H. Jin and J. Varner, "Integrins: roles in cancer development and as treatment targets," British Journal of Cancer, vol. 90, no. 3, pp. 561-565, 2004.

[18] D. Schubert, R. Dargusch, J. Raitano, and S.-W. Chan, "Cerium and yttrium oxide nanoparticles are neuroprotective," Biochemical and Biophysical Research Communications, vol. 342, no. 1, pp. 86-91, 2006.

[19] K. A. Gschneidner Jr., L. Eyring, and G. H. Lander, Handbook on the Physics and Chemistry of Rare Earths, Elsevier, Amsterdam, The Netherlands, 1978. 

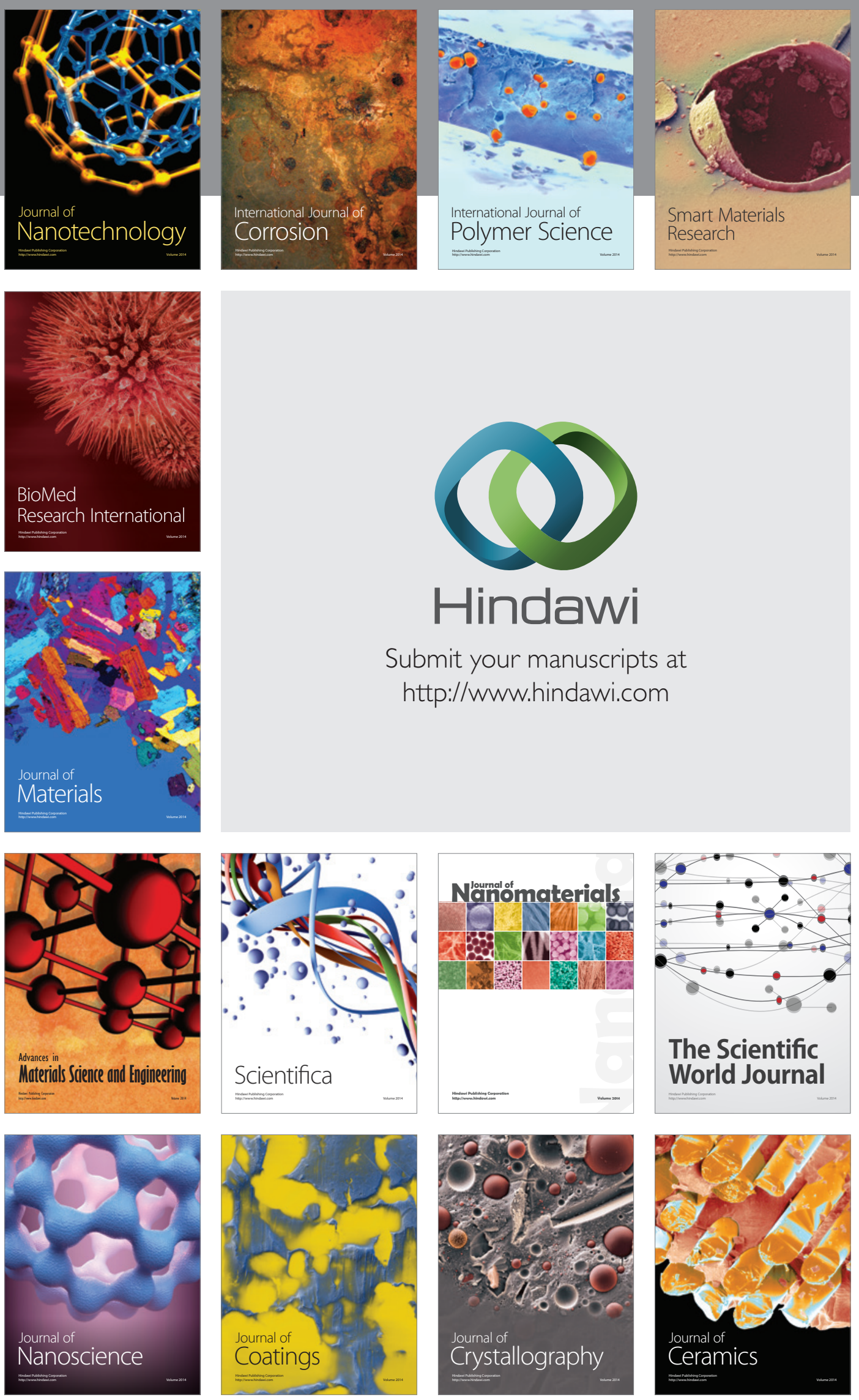

The Scientific World Journal

Submit your manuscripts at

http://www.hindawi.com

\section{World Journal}

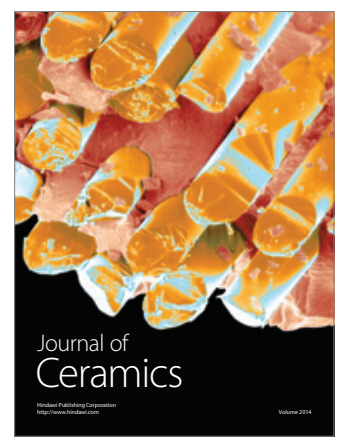

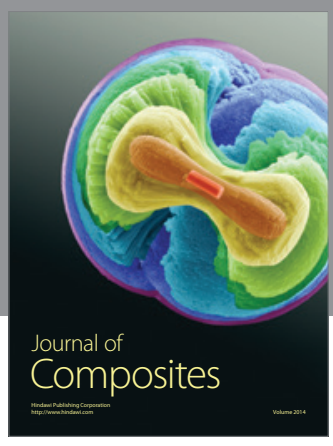
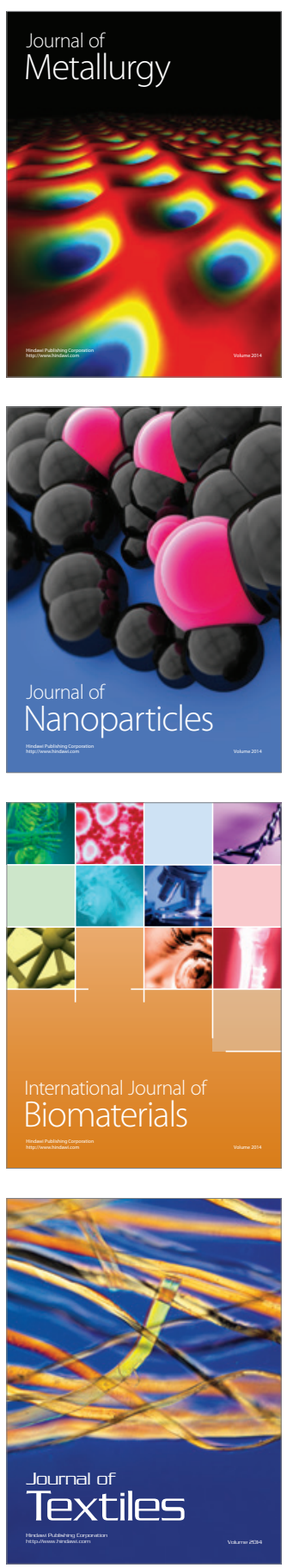